\title{
La «school readiness» e la sua misura: uno strumento di rilevazione per la scuola dell'infanzia ${ }^{1}$
}

\section{Cristina Coggi - Paola Ricchiardi}

Università degli Studi di Torino, Dipartimento di Scienze dell'Educazione e della Formazione

doi: 10.7358/ecps-2014-009-cogg cristina.coggi@unito.it paola.ricchiardi@unito.it

\section{SCHOOL READINESS AND ITS MEASUREMENT: AN ASSESSMENT TOOL FOR KINDERGARTEN}

\section{Abstract}

Many international studies have focused on school readiness. It has, therefore, become urgent to evaluate the empirical evidence around this topic. It is a complex subject, initiating a wide debate and a variety of contributions. Studies have created a deeper vision of the concept and broadened the areas that need to be researched. Moreover, the environmental conditions contributing to the development of readiness have been analyzed in combination with the possible strategies to enhance it. This contribution carries out a critical analysis of these studies. This essay is particularly focused on the aspects of the measurement of readiness and offers a review of the main tests adopted internationally to measure it. It, finally, proposes an original tool to measure cognitive readiness, suitable for Italian schools, outlining its psychometric characteristics, based on a sample of 445 children. Such a test highlights the difficulties that significant percentages of children aged between 4 and 5 have. It also allows for the evaluation of the effects of the enhancement of readiness, in order to establish the most effective ones, based on the EBE perspective.

Keywords: Kindergarten, Readiness assessment, Readiness models, Readiness test, School readiness.

1 A C. Coggi vanno attribuiti l'introduzione, i parr. 1, 3, 5, 7, 7.1, 7.2.2, 7.3, 7.4, Riferimenti bibliografici. A P. Ricchiardi vanno attribuiti i parr. 2, 4, 6, 7.2.1, Conclusione. P. Ricchiardi ha effettuato le analisi statistiche. 
Le pratiche educative possono trarre grande vantaggio da un utilizzo consapevole e critico delle evidenze di ricerca empirica sui problemi che via via emergono. Studi di ampio respiro, a livello internazionale, consentono di orientare l'azione dei professionisti su di un numero crescente di temi. Un ambito su cui esiste un grande patrimonio di ricerca empirica, specie sviluppato negli Stati Uniti, riguarda la readiness, ovvero i requisiti imprescindibili per l'ingresso nella scuola primaria. La rilevanza di tali studi è legata al fatto che l'ingresso a scuola, senza i requisiti necessari, implica un'alta percentuale di insuccesso a lungo termine. Le ricerche che si sono focalizzate sul problema hanno avuto effetti importanti anche sulle politiche scolastiche di diverse nazioni (Frank Porter Graham Child Development Center, 1999; Kiernan et al., 2008), in quanto la concezione di readiness è strettamente connessa sia con la definizione dei criteri per l'ammissione alla scuola primaria, sia con la valutazione della qualità dei programmi prescolari. Per studiare l'ampiezza degli effetti di tali programmi e l'interferenza di altri fattori, sono state realizzate sintesi degli studi empirici, anche con tecniche di meta-analisi. Queste esigono però di partire da concetti validi e condivisi di readiness e da strumenti affidabili di misura della stessa.

Nel presente contributo cercheremo di sintetizzare tali studi, per delineare l'evoluzione del concetto di readiness e per esplicitare i fattori che risultano correlati alla "prontezza scolastica». Affronteremo quindi i problemi connessi alla misura del costrutto considerato e presenteremo i test più in uso. Proporremo infine un nuovo strumento di misura degli aspetti cognitivi della school readiness, elaborato alla luce della letteratura internazionale e predisposto per i contesti italiani. Offriremo indicazioni sulle caratteristiche edumetriche dello stesso, per supportare le ricerche empiriche che intendono avvalersene. Si tratta di un sussidio adatto alla professionalità degli insegnanti, chiamati non solo ad operare diagnosi accurate, ma anche a svolgere azioni di recupero e di potenziamento delle abilità eventualmente carenti e a verificarne l'efficacia.

\section{EVOLUZIONI DEL CONCETTO DI «READINESS»}

La readiness è un costrutto multidimensionale, da tempo studiato secondo prospettive diverse. Negli Stati Uniti il termine è stato introdotto a partire dagli anni '20 del secolo scorso ed è stato oggetto di approfondimenti consistenti, soprattutto dagli anni ' 80 in poi.

Si sono succeduti nel tempo modelli differenti di interpretazione della readiness, influenzati dall'evoluzione della ricerca psicologica. Possiamo ri- 
chiamarli brevemente, anche alla luce delle rassegne di Meisels (1999) e di Andrews e Slate (2001).

Il primo modello di readiness è quello evolutivo, centrato sul bambino e sul suo sviluppo. Tale approccio considera la caratteristica in questione come l'essere pronti ad apprendere (ready to learn), in quanto maturi. Il processo di maturazione cognitiva, emotiva e psicomotoria del bambino viene interpretato come uno sviluppo spontaneo, connesso all'età. La "prontezza», in questo caso, consiste nel saper affrontare compiti adeguati all'età cronologica, definiti in maniera standard.

Il secondo modello è quello ambientale o socio-culturale. Secondo questa prospettiva, la readiness non è una caratteristica genetica, ma è una proprietà del bambino, perlopiù dipendente dalle interazioni nel suo ambiente di vita. Si tratta in altri termini di abilità che vengono apprese attraverso le prime esperienze di socializzazione.

Il terzo modello, cosiddetto delle abilità cumulative, delimita la readiness, intendendola come school readiness, ovvero come l'essere pronti per la scuola. Questa prospettiva interpreta il costrutto in esame come il possesso di prerequisiti per apprendere contenuti scolastici, grazie alla stimolazione diretta dell'adulto e alle esperienze attuate in un contesto. Secondo questa concezione, tutti i bambini sono genericamente pronti ad apprendere. Le differenze si evidenziano quando si devono affrontare specifici compiti scolastici e su questi occorre centrarsi per la diagnosi. La ricerca ha spostato dunque l'attenzione dall'essere maturi, all'essere pronti per la scuola, sottolineando la rilevanza dei concetti e delle strategie di base necessarie per l'alfabetizzazione. Quest'ultimo approccio prevede la rilevazione di abilità prescolastiche nella scuola dell'infanzia, in quanto l'apprendimento è concepito secondo un continuum progressivo che segue un iter ordinato. Secondo questa prospettiva, importanti requisiti di readiness sono i comportamenti osservabili del bambino, come riconoscere le forme e i colori o identificare oggetti, lettere e numeri.

Il quarto modello di readiness è quello socio-costruttivista, che interpreta il costrutto come l'essere pronti per apprendere, nell'interazione con gli altri. Questa prospettiva si centra sul grado in cui il bambino dimostra di saper strutturare conoscenze e capacità, nell'interazione con i pari più abili e con gli adulti, a casa e a scuola. La readiness consiste dunque nel possesso di tali requisiti, determinati culturalmente, in quanto legati alle aspettative, ai valori e alle caratteristiche del contesto in cui la scuola è inserita.

Il quinto modello, interazionista o ecologico, interpreta la readiness come una proprietà di più soggetti: bambini pronti, scuole pronte, famiglie pronte, comunità pronte, servizi pronti. Questa definizione estende lo sguardo alle interazioni multiple che il soggetto ha con l'ambiente di crescita ed offre una prospettiva utile alle politiche sociali. In quest'ottica occorre valutare la readi- 
ness di tutti gli attori coinvolti nel processo (Kagan \& Rigby, 2003). Secondo questa prospettiva:

a. le famiglie risultano "pronte» quando offrono ai bambini opportunità significative di sviluppo nei diversi ambiti (risorse ludiche, interazioni funzionali, cura, affetto, regolazione ...);

b. le comunità risultano "pronte» quando sono in grado di fornire supporti alle famiglie, specie a quelle più in difficoltà per povertà o disgregazione;

c. i servizi risultano "pronti» quando offrono interventi di qualità, accessibili a tutte le tipologie di utenti;

d. le scuole risultano «pronte» quando propongono programmi tali da garantire buone possibilità di riuscita a tutti gli alunni.

Dunque nella definizione di school readiness si è progressivamente transitati: da una visione maturativa, ad una costruttivista-sociale ed ecologica; da una concezione per tappe di sviluppo geneticamente stabilite, alla determinazione di una caratteristica in continua evoluzione, in funzione del supporto ambientale; da un'attribuzione univoca a proprietà del bambino, ad una responsabilizzazione della scuola, della famiglia e della società.

Di seguito approfondiremo alcuni aspetti della school readiness del bambino, emersi dagli studi internazionali, per passare poi ad analizzare alcune caratteristiche dei fattori ambientali di readiness. Sceglieremo gli aspetti più rilevanti in funzione delle esigenze diagnostiche e di intervento degli insegnanti.

\section{ASPETTI PERSONALI DELLA «READINESS»: ESITI DI RICERCA}

Parallelamente all'evoluzione dei modelli interpretativi della readiness, si è assistito allo sviluppo delle teorie che approfondiscono i fattori individuali connessi alla stessa. Gradualmente si è transitati da una concezione che si limitava allo sviluppo cognitivo e alle conoscenze di tipo scolastico, ad una che integra anche le abilità sociali ed emotive che favoriscono l'apprendimento. Approfondiremo dunque di seguito entrambi gli aspetti, nelle concezioni più attuali.

\section{a) Fattori cognitivi}

I fattori cognitivi, legati allo sviluppo intellettivo, riguardano, da un lato, le conoscenze prescolastiche che fondano l'alfabetizzazione, dall'altro l'intelligenza fluida, ovvero i processi connessi al problem-solving e le funzioni esecutive (Blair, 2006).

$\mathrm{Al}$ problem-solving concorrono processi quali la comprensione di una situazione complessa, la capacità di effettuare le corrette inferenze (il ragiona- 
mento), la pianificazione e la flessibilità cognitiva, l'elaborazione di più ipotesi di soluzione per affrontare la situazione problematica (pensiero creativo) e il pensiero critico, che consente di comparare le vie individuate e di selezionare quella più adeguata, eliminando quelle non idonee.

Le funzioni esecutive riguardano invece la memoria di lavoro (working memory), il controllo inibitorio e la regolazione dell'attenzione.

La memoria di lavoro permette al soggetto di ritenere e manipolare le informazioni e il loro flusso. Si tratta, per i bambini della scuola dell'infanzia, di ricordare le sequenze di una storia, l'ordine di presentazione di alcuni oggetti, le regole di un gioco e un insieme di istruzioni.

Il controllo inibitorio coinvolge la capacità di interrompere una risposta istintiva, automatica o reattiva, producendo risposte alternative tra le quali occorre scegliere, in vista degli obiettivi da raggiungere. Si tratta di una componente fondamentale dell'autoregolazione, in quanto crea un ritardo tra l'input e la risposta, consentendo così la riflessione e una scelta strategica. Il controllo inibitorio viene evidenziato dalle risposte date da un soggetto in situazione di conflitto cognitivo. Si rileva dunque se, a fronte di una provocazione o di un'alternativa molto allettante, il soggetto dimostra di saper inibire le risposte istintive e di elaborare strategie nuove. Per esempio, nell'esperimento Gift Delay Task, si chiede ai bambini di non guardare, mentre lo sperimentatore incarta rumorosamente un regalo a loro destinato (Kochanska et al., 1996). I bambini con maggior controllo riescono ad attenersi alle consegne, mentre gli altri seguono il comportamento istintuale. Altri esperimenti richiedono al soggetto di inibire le risposte automatiche, fornendo quelle richieste dallo sperimentatore: per esempio, il bambino deve digitare su una tastiera "uno» tutte le volte che il ricercatore digita «due» e viceversa; deve schiacciare il pulsante blu ogni volta che sullo schermo compare il rosso. In questo tipo di esercizi, oltre al controllo inibitorio, è coinvolta anche la working memory, nel richiamare una risposta diversa da quella spontanea. Secondo i ricercatori, ambedue i processi implicano la capacità di rappresentazione simbolica/astratta, che consente di distanziarsi dalla risposta istintiva.

Il controllo attentivo riguarda invece la capacità di mantenere l'attenzione, anche in presenza di elementi di disturbo e di spostarla in maniera mirata.

Le funzioni esecutive e i processi cognitivi si possono svolgere su diversi tipi di materiale: simbolico, iconico, comportamentale e verbale. Un aspetto rilevante dello sviluppo cognitivo è rappresentato dal patrimonio linguistico posseduto dal soggetto. Quest'ultimo è particolarmente influenzato dalle caratteristiche del contesto di crescita.

Già a 24 mesi, per esempio, un bambino che proviene da una famiglia di livello socio-culturale elevato risulta più competente di un coetaneo svantaggiato nel comprendere e pronunciare correttamente parole e frasi (Halle et 
al., 2009). Le differenze aumentano con il progredire dell'età: un bambino di contesto deprivato, a 3 anni, conosce la metà delle parole note ad un coetaneo cresciuto in un ambiente medio-alto (Hart \& Risley, 1995). Le differenze di vocabolario a 3 anni, d'altra parte, predicono gli esiti in ambito linguistico a 9 anni, come risulta da studi longitudinali, condotti su ampie coorti di studenti (Magnuson et al., 2004). A 5 anni, inoltre, un bambino di classe sociale superiore riconosce circa 22 lettere, contro le 9 identificate dai bambini svantaggiati (Howard, Grogan Dresser, \& Dunklee, 2009). Un complesso studio di metanalisi, condotto negli USA su sei ricerche longitudinali che hanno utilizzato ampi campioni, ha evidenziato che, al momento dell'ingresso a scuola, tra i fattori più predittivi del futuro successo scolastico vi sono proprio lo sviluppo linguistico e le pre-competenze legate alla lettura: conoscenza di vocaboli, riconoscimento della lettera con cui iniziano e finiscono le parole (Duncan et al., 2007). Più specificamente, secondo una meta-analisi condotta dal National Literacy Panel (Shanahan, 2005), la phonological sensitivity (ovvero la capacità di individuare e manipolare suoni e sillabe) e la conoscenza alfabetica predicono le abilità di decifrazione e comprensione della lettura. Al contrario, i bambini con ritardi nell'apprendimento del linguaggio presentano anche le maggiori probabilità di incontrare difficoltà nella decifrazione delle parole e nella comprensione della lettura (Schuele, 2004).

\section{b) Fattori socio-emozionali}

Numerosi studi hanno supportato, con dati empirici, l'ipotesi che, a fianco delle variabili cognitive, abbiano un ruolo rilevante, per determinare la readiness scolastica, i fattori socio-emozionali, quali la motivazione all'apprendimento, la fiducia in sé, il contenimento dell'ansia, la regolazione delle emozioni in senso prosociale e le relazioni positive con i pari e con gli insegnanti (Ladd, Kochenderfer, \& Coleman, 1997). La motivazione ad apprendere, in particolare, risulta una condizione preliminare per avviare la strutturazione di conoscenze e competenze. Il desiderio di conoscere è inoltre fondamentale per sostenere lo sforzo cognitivo e la perseveranza (Deci \& Ryan, 1985). La motivazione è connessa con altre variabili, quali l'autostima e la percezione di competenza. Gli aspetti emotivi risultano correlati inoltre con lo sviluppo linguistico e con le abilità di comunicazione con i pari e con l'adulto, facilitando l'acquisizione di nuove competenze (La Paro \& Pianta, 2000).

Secondo le evidenze di ricerca, l'adattamento socio-emozionale predice, in modo importante, il successo scolastico dei soggetti, a parità di potere cognitivo e risorse familiari (Ladd \& Burgess, 1999). I bambini che hanno maggiori probabilità di avere successo nel passaggio dalla scuola dell'infanzia alla scuola primaria, sono quelli in grado di: identificare le emozioni proprie e altrui; relazionarsi con insegnanti e coetanei in maniera positiva; gestire 
sentimenti di rabbia, di frustrazione e angoscia di fronte a situazioni emotivamente stressanti; mostrare motivazione all'apprendimento; lavorare concentrati e cooperare in un'attività strutturata (Raver \& Knitze, 2002).

Risultano invece indicatori di disadattamento socio-emozionale, che consentono di prevedere frequentemente l'insuccesso scolastico: l'aggressività; l'incapacità di controllare i comportamenti impulsivi; l'incapacità di prestare attenzione ai compiti o di interpretare le indicazioni; la messa in atto di comportamenti oppositivi; l'incapacità di collaborare con gli altri; la ricerca continua di attenzione da parte di coetanei e insegnanti o al contrario l'evitamento di pari o di adulti.

Nell'ambito di una ricerca, condotta su 356 bambini di quattro anni (Bierman et al., 2009), è stata calcolata la correlazione tra gli aspetti cognitivi di readiness (distinguendo tra conoscenze scolastiche e funzioni esecutive) e gli aspetti comportamentali e affettivi della stessa (quali la partecipazione in classe, il comportamento prosociale e regolato). Dall'indagine emerge che la partecipazione in classe e il comportamento prosociale risultano correlati positivamente sia con lo sviluppo di conoscenze scolastiche, sia con quello delle funzioni esecutive.

\section{AMBIENTE SOCIO-CULTURALE E FATTORI DI RISCHIO PER LA «SCHOOL READINESS»: STUDI EMPIRICI}

I fattori ambientali che le ricerche hanno associato più frequentemente a ritardi nella school readiness riguardano: la deprivazione economica e culturale del contesto di vita del bambino; le caratteristiche della famiglia; la qualità dell'offerta formativa nella scuola dell'infanzia; il tipo di supporto della comunità di appartenenza.

Per quanto riguarda la famiglia, sono stati studiati: il reddito familiare; il livello culturale dei genitori (specie materno); l'occupazione di padre e madre; la composizione, la stabilità e alcune caratteristiche del nucleo familiare (famiglia monoparentale, numero di fratelli, età della madre, separazioni, divorzi e ricomposizioni del nucleo); la lingua parlata in famiglia; l'essere inclusi o meno nel circuito dell'assistenza. In generale la povertà continua a risultare un fattore di rischio per lo sviluppo psico-sociale del bambino. Sono stati elaborati, a questo proposito, due modelli teorici che cercano di spiegare perché le carenze economiche incidono sulla school readiness nell'infanzia.

Si tratta da un lato del family stress model, che attribuisce al tipo di relazioni ed interazioni familiari, che caratterizzano i contesti finanziariamente svantaggiati, gli effetti negativi sui comportamenti dei soggetti in 
crescita. Tale modello spiega bene le relazioni tra povertà, aspetti emotivi e comportamentali del minore, illustrando come lo stress familiare, generato da condizioni economiche precarie, ostacoli un'adeguata maturazione sociocomportamentale e di autoregolazione del bambino. Infatti, i minori che vivono con adulti incapaci di regolare le proprie emozioni e di risolvere $\mathrm{i}$ conflitti senza ricorrere a violenze, sperimentano spesso sentimenti negativi, come rabbia, depressione, ansia (Sylva, 2002). Queste situazioni si verificano più di frequente quando la madre, rimasta sola, non può usufruire di un supporto sociale. In tal caso la donna risulta abitualmente più punitiva $\mathrm{e}$ restrittiva con i bambini (Bornstein, 1995), meno capace di ascoltare, di interagire verbalmente e di stimolare il problem-solving sociale dei figli. Anche la depressione materna ha effetti pesanti sullo sviluppo intellettuale, linguistico e socio-emozionale dei bambini (Field, 1995).

Il secondo modello, l'investment model, illustra gli effetti, a breve e a lungo termine, delle carenze di cure parentali, di stimoli cognitivi, affettivi e relazionali, di risorse ludiche ed ambientali, capaci di arricchire l'esperienza e di favorire l'elaborazione cognitiva e linguistica dei bambini (Duncan \& Magnuson, 2005). I minori che vivono in ambienti malsani e poco stimolanti per la crescita hanno un patrimonio di conoscenze, in linea con quelle scolastiche, molto limitato. Sono inoltre più a rischio perché hanno frequentemente genitori molto giovani, inesperti, con relazioni instabili e/o con problemi di salute fisica o mentale, con livelli culturali bassi. Anche l'ambiente in cui la famiglia risiede può incidere negativamente, in quanto abitualmente le famiglie deprivate vivono in contesti ad alto livello di criminalità, con maggiori rischi per la vita, minori possibilità di cura e prevenzione sanitaria per i bambini.

Un'indagine australiana ha mostrato però che abitualmente non è solo la povertà di mezzi economici ad impedire alti livelli di school readiness, ma piuttosto il cumulo di fattori di rischio connesso alla povertà. La ricerca documenta che nel $60 \%$ dei casi, le famiglie povere presentano almeno 4 fattori di rischio contemporaneamente e nel 10\% fino a 7 fattori (Edwards et al., 2009). È dunque l'esposizione ai rischi multipli connessi alla povertà che moltiplica nei bambini carenze di readiness scolastica (Webster-Stratton, Reid, \& Stoolmiller, 2008).

Nella stessa linea, altri studi distinguono il semplice svantaggio socioeconomico dallo stato di povertà vero e proprio, connesso con rischi multipli. Il solo svantaggio economico non necessariamente incide sulla readiness, specie negli aspetti socio-emozionali. Lo stato di povertà risulta invece collegato a incapacità di regolazione emozionale, a scarse abilità sociali e a maggiori problemi di condotta, che persistono anche nei livelli scolastici successivi alla scuola dell'infanzia, come attestano diversi studi longitudinali 
(Huffman, Mehlinger, \& Kerivan, 2001). Secondo le ricerche, ben un quarto dei bambini in condizioni di povertà presenta i problemi descritti (Keenan et al., 1997). Per questi soggetti uno degli elementi più importanti di protezione è rappresentato dall'incontrare almeno un docente supportivo, capace di adottare una disciplina non rigida, di mettere in pratica strategie proattive di insegnamento e di incoraggiare adeguatamente i progressi degli allievi (Pianta \& Walsh, 1998).

Un caso specifico è costituito dalle famiglie migrate, che presentano di frequente numerose condizioni di svantaggio. I loro bambini, manifestano, di conseguenza, solitamente livelli di school readiness inferiori rispetto a quelli dei coetanei con genitori autoctoni. Non si può ignorare, a questo proposito, l'effetto negativo dello sradicamento da migrazione e l'incidenza della discontinuità tra la cultura familiare e le richieste scolastiche (Hibel, 2009). Anche gli stereotipi incidono negativamente sull'integrazione scolastica e sulla riuscita di alcune etnie. Le ricerche hanno messo in luce però che la distanza tra autoctoni e migrati è meno evidente se si confrontano gruppi di pari livello socio-culturale, confermando l'incidenza rilevante del fattore povertà (Portes \& Rumbaut, 2000).

Secondo ricerche americane, i bambini svantaggiati e quelli immigrati frequentano inoltre meno regolarmente la scuola dell'infanzia e afferiscono con maggiore probabilità ad istituti che si trovano in quartieri deprivati e hanno un'offerta formativa di minor qualità (Magnuson et al., 2004). Nei quartieri ghetto si concentrano infatti frequentemente le scuole con maggiori problemi: alunni poco sostenuti dalle famiglie, insegnanti demotivati e meno formati, condizioni organizzative inadeguate e risorse economico-culturali scarse. Gli esiti complessivi in tali istituti risultano dunque di basso livello. Si tratta di un problema che riguarda tutte le fasce d'età, da quella prescolastica a quella scolastica. L'opportunità invece di fruire precocemente di un'offerta formativa di qualità costituisce uno dei maggiori fattori di promozione della readiness (Loeb et al., 2007), specie per i soggetti che hanno condizioni di vita ipostimolanti a casa (McCartney et al., 2007). In particolare, costituiscono elementi di qualità della didattica: la realizzazione di attività in piccolo gruppo, l'incoraggiamento verbale, la facilitazione nei confronti dell'acquisizione di nuove conoscenze e comportamenti, l'utilizzo frequente della narrazione e della lettura (Dickinson \& Tabors, 2001) e uno stile relazionale accogliente e valorizzante dell'adulto (Tran \& Weinraub, 2006).

Un ulteriore esempio di come l'ambiente interagisca con le caratteristiche individuali, favorendo o meno la readiness, riguarda le differenze di genere. Alcune ricerche hanno evidenziato che le diversità nella preparazione alla scuola tra maschi e femmine, sono a favore di queste ultime e appaiono legate all'interazione tra fattori biologici e culturali. La lateralizzazione del 
linguaggio, anticipata nelle bambine (Bornstein, Hahn, \& Haynes, 2004), porterebbe i caregiver a stimolarle maggiormente nella comunicazione, avvantaggiandole ulteriormente (Chaplin, Cole, \& Zahn-Waxler, 2005). La scuola dell'infanzia, a sua volta, abitualmente richiede tempi di attenzione e propone setting didattici più adatti agli stili cognitivi delle bambine piuttosto che a quelli dei maschi (Zill \& West, 2001), incrementando ulteriormente le distanze tra i livelli medi di genere.

\section{SCOPI, MODELLI E STRUMENTI DI RILEVAZIONE DELLA «SCHOOL READINESS» DELL'ALUNNO}

Misurare la readiness è un'esigenza che può derivare da scopi diversi. Tra le intenzioni si possono distinguere: controllare la percentuale dei bambini pronti per la scuola, a livello nazionale o a livello locale; selezionare i bambini pronti ad accedere alla scuola; informare i genitori e gli insegnanti sul livello individuale raggiunto; monitorare le popolazioni a rischio ed identificare i soggetti che necessitano di un recupero; pianificare adeguati interventi; valutare l'efficacia dei programmi per l'infanzia, delle strategie didattiche adottate e degli stili di accudimento genitoriali nel favorire la readiness; fornire informazioni per i decisori politici.

A seconda dello scopo e degli ambiti toccati variano sia gli strumenti sia le fasce d'età su cui avviene la rilevazione ${ }^{2}$.

Gli strumenti di readiness si possono classificare anche in base al modello a cui fanno riferimento. Sceglieremo quest'ultimo criterio per descrivere alcuni tra i test più utilizzati in ambito anglofono. Lo scopo non è quello di una rassegna sistematica, vista l'ampia produzione esistente, ma di mostrare come sono evoluti i criteri di scelta delle modalità di misura ${ }^{3}$.

\section{a) Misure di «readiness» secondo il modello evolutivo}

Tra gli strumenti frequentemente utilizzati per rilevare le tappe di sviluppo del soggetto, occorre citare il Gesell Readiness Test (1947), che valuta i comportamenti motori, lo sviluppo linguistico e l'adattamento sociale. Nella medesima categoria si collocano i Piagetian Tests (Kenny, 1978), trasposizione delle esperienze piagetiane per individuare gli stadi cognitivi di sviluppo. Tali 5 anni.

2 Abitualmente negli Stati Uniti le valutazioni di readiness riguardano i bambini dai 3 ai

3 Tra le rassegne sistematiche si possono annoverare quella di Niemeyer e Scott-Little (2001), che hanno individuato 29 strumenti, e quella di Garber (2007), che annovera 39 strumenti tra i più utilizzati. 
test rilevano i gradi di acquisizione delle operazioni logiche, della reversibilità e della conservazione, della seriazione, dei concetti riferibili allo spazio e al numero (Boncori, 1993). Ancora riconducibile al modello evolutivo è il Test di Woodcock-Johnson: si tratta di un insieme di test di intelligenza sviluppato nel 1977 da R. Woodcock e M.E. Bonner Johnson, con revisioni nel 1989 e nel 2001 (Woodcock-Johnson III). Le aree indagate riguardano: la comprensione e la conoscenza; il recupero a lungo termine; il pensiero visivo-spaziale; l'elaborazione uditiva; il ragionamento fluido; la velocità di elaborazione; la memoria a breve termine; la conoscenza di quantità e la letto-scrittura. Il test determina una capacità intellettiva generale.

\section{b) Misure di «readiness» secondo il modello ambientale e delle abilità cumulative: test standardizzati}

Aspetti generali. - Alcuni strumenti sono focalizzati maggiormente su concetti e abilità preliminari agli apprendimenti scolastici. Per esempio, il Metropolitan Readiness Tests (Nurss \& McGauvran, 1976) indaga aspetti come la conoscenza del lessico di base in uso a scuola, la capacità di ascolto, l'individuazione delle lettere iniziali dei termini, la corrispondenza suono-lettera, la capacità di individuare rime, il riconoscimento di lettere, il concetto di quantità, le operazioni, la capacità di trascrizione, la memoria uditiva. Una parte specifica nei diversi test di readiness viene dunque riservata alla diagnosi delle competenze di pre-lettura e pre-scrittura, a cui si aggiungono le rilevazioni delle preconoscenze matematiche. Ancora più incentrato sulle conoscenze prescolastiche risulta il Bracken School Readiness Assessment (Bracken, 2007), che si focalizza sulla conoscenza di forme, colori, lettere e numeri; sul conteggio; sull'eduzione di relazioni tra grandezze e sulle comparazioni.

Uno strumento volto a valutare aspetti percettivi e grafici utili a scuola è il Who am I? (De Lemons \& Doing, 2000), che rileva: la capacità di riprodurre figure (cerchio, croce, quadrato, triangolo, diamante), di operare con simboli (scrivendo il proprio nome, numeri, lettere, parole, frasi) e di colorare.

Diagnosi su aspetti specifici. - Diversi test hanno approfondito una singola area di abilità, predittiva della riuscita nella letto-scrittura $o$ in matematica.

Per valutare le capacità ricettive del linguaggio, si può citare il Peabody di L.M. Dunn e D.M. Dunn (2000), pubblicato per la prima volta nel 1959 e adottato diffusamente negli USA.

Un ambito particolarmente studiato è la consapevolezza fonologica, che risulta fortemente connessa con le abilità di decodifica nella lettura (Gibbs, 2004). Si tratta della conoscenza che il soggetto ha della struttura fonetica delle parole e della capacità di identificare, confrontare, separare, combinare e generare suoni. Fa parte dei processi metalinguistici, in quanto richiede di riflettere sulla lingua e di esercitare competenze fonologiche, come il discri- 
minare suoni e memorizzarli. Il Phonological Awareness Test (Robertson \& Salter, 1997), per esempio, richiede il riconoscimento dei suoni, la segmentazione di parole in sillabe e fonemi.

Altri strumenti ancora si focalizzano su singoli processi cognitivi considerati rilevanti per la readiness. Si tratta di prove che misurano la memoria di lavoro, le capacità attentive e il controllo inibitorio. Il Test di Davis e Pratt (1996) rileva la working memory, richiedendo ai bambini di ripetere una lista di parole in senso contrario a quello ascoltato. Un altro strumento che valuta la working memory e il controllo inibitorio è il Peg Tapping Task, il quale richiede al bambino di colpire due volte un piolo quando lo sperimentatore lo tocca una volta e viceversa (Diamond \& Taylor, 1996). Gli strumenti volti a rilevare il medesimo costrutto sono numerosi. Per esempio, il Wisconsin Card Sorting Test (Grant \& Berg, 1948) presenta ai bambini una serie di carte che differiscono per forma, colore e numero e propone loro di abbinarle, secondo una determinata regola. In seguito richiede di cambiare la regola di abbinamento. I bambini con bassi livelli nelle funzioni esecutive continuano ad abbinare le carte seguendo la regola precedente. Lo strumento evidenzia eventuali carenze di flessibilità, che comportano poi difficoltà nel problem-solving. In forma analoga sono stati elaborati test come il più recente Dimensional Change Card Sort (Frey \& Zelazo, 2003), finalizzato ad accertare le funzioni esecutive in situazioni di compito progressivamente più complesse per l'introduzione di nuove regole di classificazione, che tengono conto di più criteri.

Un test per valutare indirettamente le funzioni esecutive nei bambini dai 5 anni in su è il Behavior Rating Inventory of Executive Function (Gioia et al., 2000). Si tratta di questionari che possono essere compilati da insegnanti e genitori e riguardano il controllo inibitorio, lo spostamento dell'attenzione e il monitoraggio della stessa.

$\mathrm{Vi}$ sono strumenti poi che valutano in specifico gli aspetti socio-emotivi di readiness. Un esempio, può essere lo Strenghts and Difficulties Questionnaire (SDQ). Si tratta di un breve questionario, rivolto a insegnanti e genitori per valutare le competenze sociali dei bambini dai 3 anni in su. Comprende cinque aree: manifestazioni emozionali, problemi di comportamento, iperattività e disattenzione, rapporti problematici con i pari, comportamento prosociale (Goodman, 1997 e 2001).

\section{c) Strumenti di «readiness» secondo il modello socio-costruttivista}

Gli autori socio-costruttivisti hanno realizzato misure che coinvolgono le comunità. Queste si impegnano a definire le dimensioni rilevanti per la readiness dei bambini nel proprio contesto, con indicatori positivi e negativi (Love, Aber, \& Brooks-Gunn, 1994). Le misure portano a profili collettivi di «maturità scolastica», secondo la comunità di appartenenza. 
In questo approccio le misure devono essere realizzate utilizzando più strumenti, e adottando prospettive differenti: nella maggior parte degli Stati viene utilizzato un insieme ampio di sussidi.

\section{d) Strumenti di «readiness» secondo il modello interazionista ed ecologico}

Secondo questo modello, le rilevazioni vanno condotte mantenendo il focus contemporaneamente sul bambino e sul contesto.

Gli autori che seguono questo approccio propongono strumenti per valutare la readiness del bambino, della scuola e della comunità. Ci soffermeremo in particolare sugli strumenti adottati per il bambino. Tra gli strumenti di osservazione sistematica si può citare l'High/Scope Preschool Child Observation Record (COR; Brickman \& Barton, 2003). Lo strumento indaga l'iniziativa, le relazioni sociali, la creatività, le abilità motorie e le attitudini musicali, il linguaggio, la literacy, le competenze in matematica e scienze. Uno strumento specifico, che si aggiunge a tale scala di osservazione, è ELSA (The Early Literacy Skills Assessment), che si colloca in una logica di valutazione autentica ed analizza le competenze dei bambini attraverso l'interazione con storybook. Uno strumento elaborato e diffuso invece in Canada è l'EDI (The Early Development Instrument, Janus, 2007), applicato anche in Australia (come AEDI). Lo strumento è rivolto a bambini dai 5 ai 7 anni. La scala di osservazione si suddivide nelle seguenti aree: salute e benessere del bambino; competenze sociali; abilità linguistiche; sviluppo delle strategie comunicative e delle conoscenze generali.

Le misure dell'EDI dal 2003 vengono affiancate da quelle derivanti dal Kindergarten Parent Questionnaire. Si tratta di uno strumento rivolto ai genitori, che riguarda la salute e lo sviluppo del bambino, la sua cura, i dati relativi all'andamento nel nido e nella scuola dell'infanzia, le informazioni sulla famiglia e sull'ambiente di vita.

\section{CRITICHE ALLE MISURE DI «READINESS» E AL LORO UTILIZZO}

Alcuni autori sottolineano l'importanza di utilizzare con prudenza le misure di school readiness (Maxwell \& Clifford, 2004), in quanto possono derivare da strumenti con diverso livello di validità e fedeltà. Alcuni di questi sussidi, come i questionari aperti rivolti ai genitori, risultano utili per dare indicazioni e pianificare adeguatamente la didattica, ma non sono sufficienti per un approfondimento clinico. In molti casi, specie negli USA, sono stati utilizzati invece gli screening di readiness per identificare gli alunni con disabilità, senza adottare strumenti adeguati allo scopo (Keating, 2007). Un altro rischio 
è di cristallizzare per lungo tempo la valutazione negativa, senza intervenire adeguatamente. Le attese negative nei confronti di bambini che mostrano un ritardo in una fase specifica della crescita, spiegabile anche con fattori accidentali, rischiano di ostacolare ulteriormente il loro recupero.

Un ulteriore rischio è quello di effettuare inferenze a partire da un solo strumento, che non può rendere conto adeguatamente della multifattorialità del costrutto.

Alcuni autori sottolineano anche che esiste una grande variabilità nelle abilità dei bambini, per cui le performance dei piccoli sono multidimensionali, episodiche e culturalmente e contestualmente influenzate (Kagan, Moore, \& Bredekamp, 1995; De Feyter \& Winsler, 2009).

\section{COME VAlUTANO SPONTANEAMENTE LA «READINESS» GLI INSEGNANTI?}

Gli insegnanti della scuola dell'infanzia, avendo l'opportunità di un contatto costante con i bambini per diversi anni, possono effettuare valutazioni dettagliate sulle loro capacità e progressi. Resta da accertare la validità di tali rilevazioni, quando non sono supportate da strumenti adeguati.

A questo proposito la Carnegie Foundation for the Advancement of Teaching (National Center for Education Statistics, 1993) ha realizzato, nel 1991 un'indagine che ha coinvolto più di 20.000 insegnanti di 50 Stati per rilevare gli indicatori di readiness utilizzati abitualmente dai docenti di scuola dell'infanzia. Gli insegnanti hanno collocato ai primi tre posti: la salute fisica e l'adeguata nutrizione; la capacità di comunicare verbalmente bisogni, desideri e pensieri; l'entusiasmo e la curiosità nell'affrontare nuove attività. Per gli aspetti sociali, gli insegnanti ritengono indicatore importante di readiness il saper comunicare oralmente, rispettando il proprio turno di parola. Le caratteristiche considerate meno importanti dai rispondenti sono state invece quelle più legate al curricolo di prealfabetizzazione e all'intelligenza generale: le abilità di problem-solving; la capacità di identificare i colori primari e le forme geometriche regolari; la capacità di utilizzare penne e pennarelli; la conoscenza dell'alfabeto e la capacità di contare fino a venti. I docenti afroamericani, nella medesima indagine, danno invece meno importanza agli aspetti sociali di readiness e più rilevanza alle competenze prescolastiche formali. Lo studio evidenzia dunque discordanze tra gruppi diversi di docenti, in base all'etnia.

Una ricerca successiva (Mashburn \& Henry, 2004), condotta al fine di verificare l'affidabilità degli insegnanti nel valutare la readiness, ha compa- 
rato le valutazioni di insegnanti di livello scolastico differente (nido, scuola dell'infanzia) e le ha messe in relazione con rilevazioni oggettive, condotte da ricercatori su un campione di 655 bambini. Lo studio ha evidenziato che entrambi i gruppi di insegnanti presentano un certo accordo con i ricercatori nel valutare le abilità di base, come denominare i numeri o contare. Divergono invece tra loro e con i ricercatori nella valutazione di abilità complesse, come quelle di problem-solving o quelle linguistiche. Le valutazioni degli educatori del nido sono risultate meno valide di quelle degli insegnanti della scuola dell'infanzia. La validità aumenta in relazione ai livelli di formazione degli insegnanti: i docenti con più anni di formazione, risultano più affidabili.

Gli studi sottolineano inoltre che gli insegnanti possono fornire valutazioni più adeguate se dotati di strumenti validi e affidabili. Presenteremo dunque di seguito un sussidio realizzato per consentire ai docenti italiani di individuare la "cognitive readiness» dei loro alunni negli ultimi due anni della scuola dell'infanzia. Ne descriveremo inoltre le qualità edumetriche.

\section{UNO STRUMENTO PER LA «COGNITIVE READINESS»}

A fronte della carenza di strumenti di misurazione della readiness a disposizione degli insegnanti italiani, è stato predisposto dalle autrici di questo contributo un kit di Prove di readiness scolastica finalizzate a rilevare la readiness cognitiva dei bambini di 4-5 anni. Il test presenta due forme (forma I - 4 anni e forma II -5 anni), che valutano i medesimi processi con due livelli di complessità differenti, adatti rispettivamente ai 4 e ai 5 anni. Lo strumento consente dunque di effettuare rilevazioni in tempi differenziati e di cogliere anche l'evolvere dei profili individuali. Gli studi hanno infatti evidenziato come punto di debolezza delle rilevazioni di readiness la natura episodica delle stesse. Alla luce degli studi sulla school readiness, che hanno individuato tra i maggiori predittori del successo scolastico i processi cognitivi di problem-solving e le funzioni esecutive connesse (memoria di lavoro, controllo inibitorio, monitoraggio dell'attenzione), si è deciso di realizzare uno strumento focalizzato principalmente su tali aspetti. Lo strumento può essere collocato all'interno della rassegna presentata, tra quelli che valutano aspetti cognitivi rilevanti per l'apprendimento scolastico, non direttamente connessi alla pre-alfabetizzazione, per la quale esistono già altri strumenti (Zanetti \& Miazza, 2003).

Le prove da noi realizzate per la scuola dell'infanzia italiana, valutano il livello conseguito dai bambini nei processi cognitivi di base (conoscenza, 
memoria, eduzione di relazioni, ragionamento) e superiori (capacità critica e creatività), utili per il problem-solving. Si tratta di processi individuati dal modello di J.P. Guilford, rivisto secondo gli studi di Anderson e Krathwohl (Coggi \& Ricchiardi, 2009) e arricchito con gli apporti recenti della psicologia dello sviluppo sulle funzioni esecutive (Blair, 2006).

Lo strumento propone situazioni ludiche per favorire il massimo coinvolgimento dei bambini e utilizza schede e materiali di gioco.

\subsection{Contenuti dello strumento}

Lo strumento si articola in 8 scale per rilevare i diversi processi cognitivi, facendo operare l'intelligenza su materiale iconico e verbale. Gli esercizi vengono proposti all'interno di una cornice ludica diversa per ciascun esercizio.

La somministrazione individuale richiede circa 30 minuti per la forma I e 40 per la forma II.

La prime domande coinvolgono la memoria di lavoro e chiedono al bambino di ricordare serie di immagini (memoria visiva) e sequenze di frasi in rima (memoria verbale).

La sezione successiva della prova si propone di testare il bagaglio di conoscenze dei bambini, campionando tra quelle più stimolate nell'infanzia (nomi di animali e dei loro ambienti, di frutta e verdura). Il bambino deve denominare correttamente le figure proposte dal somministratore.

La terza sezione (comprensione) è la più ampia e comprende innanzitutto l'eduzione di relazioni tra concetti semplici (concetti spaziali, quali sopra, sotto; grande, piccolo; giovane, anziano; caldo, freddo ...; relazione tra mamma e piccolo; tra professionista ed un oggetto della professione). Segue la rilevazione delle capacità di ordinamento, prima per grandezza (es. sequenza di animali di varia dimensione) e poi sulla base di un criterio temporale (es. sequenzializzazione delle fasi utilizzate da un soggetto per vestirsi). Si chiede quindi ai bambini di edurre relazioni e classificare oggetti, per forma e colore e in base alla categoria di appartenenza (fiori, animali ...), giungendo anche ad applicare contemporaneamente due criteri o la negazione.

La quarta sezione riguarda il ragionamento, richiede dunque l'inferenza a partire da quesiti semplici. Per esempio, mostrando all'intervistato tre fotografie di soggetti femminili di diversa età, si domanda quale potrebbe essere la mamma di una bambina di tre anni.

La quinta sezione riguarda la capacità critica applicata a materiale figurativo: richiede di individuare l'errore o l'assurdità in un'immagine e l'intruso in un insieme di oggetti. 
La sesta sezione intende testare la creatività con materiale verbale: si richiede di completare una storia. Tale quesito esige però un adeguato bagaglio conoscitivo e lessicale. La creatività viene valutata in seguito anche con materiale figurativo, attraverso la costruzione di pupazzi differenti a partire da un insieme di pezzi.

L'ultima sezione riguarda il conteggio di diversi oggetti.

\subsection{Validità e affidabilità della prova}

Le caratteristiche edumetriche della prova sono state verificate su un campione piemontese ragionato di 445 soggetti di 4-5 anni (di cui 111 di 4 anni e 334 di 5 anni) di livello socio-culturale differenziato. Illustreremo di seguito in forma sintetica le misure di validità e fedeltà.

\subsubsection{Validità}

Face validity. - Per rilevare la face validity, la prova è stata sottoposta al vaglio di 10 psicologi dello sviluppo che hanno valutato l'utilità e l'adeguatezza dei quesiti. Dalle valutazioni emerge che la prova risulta molto utile per l'82,5\% degli item.

Per quanto riguarda l'adeguatezza gli item si collocano prevalentemente tra "molto» e «abbastanza» adeguato. Le difficoltà derivano principalmente da problemi grafici e da alcune consegne che non risultavano totalmente chiare nella prima versione della prova.

Validità di costrutto. - È stata effettuata l'analisi fattoriale per rilevare la validità di costrutto delle prove. L'analisi (condotta con il metodo Varimax, con matrice ruotata) spiega complessivamente il $72,55 \%$ della varianza, con l'estrazione di due fattori. Il primo spiega il $37,17 \%$ della varianza e il secondo il $35,37 \%$ (vd. Tabella 1). Tutti gli item hanno una saturazione fattoriale di almeno 0,5 in una delle due componenti.

La prima componente raggruppa le conoscenze prescolastiche. Si tratta del lessico di base per la scuola dell'infanzia $(0,704)$ e del conteggio entro il $12(0,710)$. Include anche i processi cognitivi superiori (il ragionamento con lo 0,723 ; la capacità critica con 0,905 ; la creatività con 0,686 ), esercitati prevalentemente su materiale verbale.

La seconda componente comprende: la working memory, esercitata sia su materiale visivo $(0,613)$ che verbale $(0,490)$ e tre elementi che afferiscono alla comprensione: l'eduzione di relazioni semplici $(0,922)$, l'ordinamento di sequenze $(0,612)$ e la classificazione $(0,945)$. 
Tabella. 1. - Analisi fattoriale.

\begin{tabular}{lcc}
\hline & \multicolumn{2}{c}{ Componenti } \\
& 1 & 2 \\
\hline Memoria visiva & &, 613 \\
Memoria verbale & &, 490 \\
Conoscenza &, 704 & \\
Eduzione di relazioni & &, 922 \\
Ordinamento & &, 612 \\
Classificazione & &, 945 \\
Ragionamento &, 723 & \\
Capacità critica &, 905 & \\
Creatività &, 686 & \\
Conteggio &, 710 & \\
\hline
\end{tabular}

Validità concorrente. - Lo strumento, in una prima versione, ha mostrato validità concorrente con il test LAP (Learning Accomplishement Profile di Sanford $\&$ Zelman, 2003), rispetto alla scala delle abilità cognitive e del linguaggio.

L'indice di correlazione $r$ di Pearson, calcolato su 60 casi, è risultato 0,70 , significativo con $\mathrm{p}<0,01$.

Item analysis. - Il calcolo degli indici di difficoltà rivela che i quesiti sono in buona parte adeguati all'età dei bambini. Sono infatti quasi tutti compresi tra 0,25 e 0,75 (vd. Tabelle 2 e 3 ).

Tra i quesiti che escono da questo range, risultando particolarmente facili, ci sono quelli relativi alla classificazione sia per i 4 anni (indice di difficoltà $=0,87$ ) che per i 5 anni (indice di difficoltà $=0,78$ ). Per i 5 anni risulta facile anche l'area del conteggio con numeri entro il 12 (indice di difficoltà $=0,77$ ).

Tali aree sono state mantenute nella prova in quanto essenziali per la readiness scolastica, ma le domande sono state rese più complesse in seguito alle analisi ed è stata attualmente avviata una nuova validazione della prova rivista. Risultano complesse, benché con indice di difficoltà superiore allo 0,25 , le prove di capacità critica e creatività. Si tratta di processi cognitivi superiori, spesso poco stimolati nella scuola dell'infanzia.

Gli esercizi di working memory, sia applicati a materiale visivo che verbale, risultano di complessità media per la fascia di popolazione a cui è stato somministrato il test (indice di difficoltà $=0,57$ per i 4 anni; indice di difficoltà $=0,49$ per i 5 anni). 

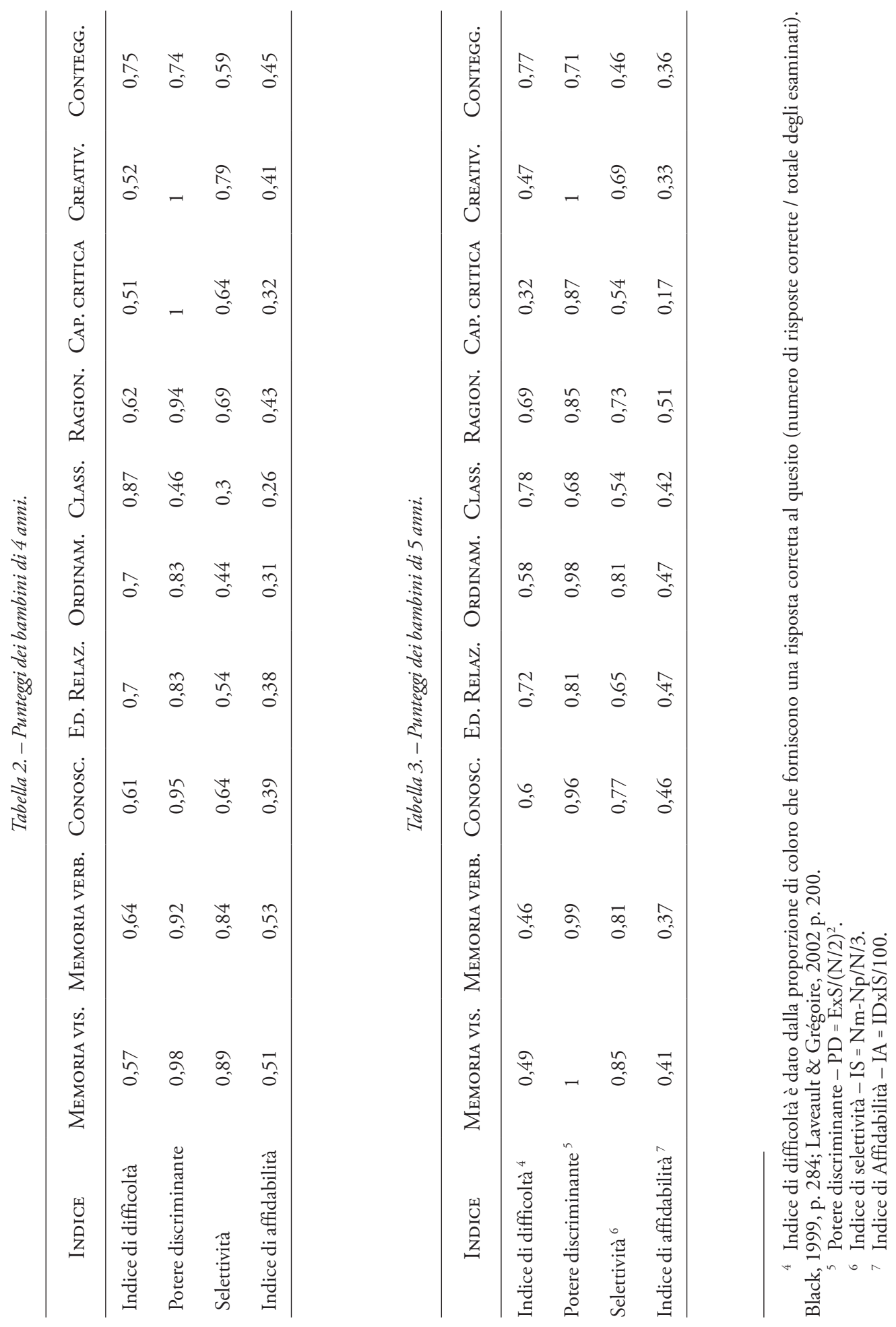
Riportiamo nelle Tabelle 2 e 3 gli esiti dell'item analysis che abbiamo commentato. 0,70 .

Tutti gli item presentano un buon potere discriminante, superiore allo

Per i quattro anni gli item che discriminano meglio sono quelli di «creatività» e "capacità critica», seguiti da quelli relativi a "memoria visiva» e "conoscenza».

Per la prova rivolta ai bambini di cinque anni, le aree maggiormente capaci di discriminare gli alunni migliori da quelli più in difficoltà risultano nuovamente la «creatività» e la «memoria visiva», seguite dalle domande di «memoria verbale», «ordinamento» e «conoscenza».

Gli item meno discriminanti riguardano la "classificazione», per ambedue le prove, in quanto le domande risultano molto semplici, come si è detto.

Gli item più selettivi sono quelli relativi alla memoria sia per la forma I -4 anni che per la forma II -5 anni.

Gli item più affidabili risultano essere quelli di «memoria» per i quattro anni e quelli di «ragionamento» per i cinque anni.

\subsubsection{Attendibilità}

Alfa di Cronbach. - L'affidabilità è stata accertata, calcolando l'indice alfa di Cronbach, sia sulla versione per i 4 anni che su quella per i 5 anni.

Per i 4 anni l'alfa è pari allo 0,806 , mentre per i 5 anni è pari allo 0,872 . Si evidenzia dunque una buona affidabilità del test per ambedue le fasce d'età, in quanto l'indice è superiore in entrambi i casi allo 0,80 .

Test-Retest. - Somministrando a distanza di quattro mesi la prova per verificare la stabilità delle rilevazioni su un campione di 26 soggetti si ottiene un indice di correlazione tra i punteggi ( $\mathrm{r}$ di Bravais-Pearson) pari a 0,87, significativo con $\mathrm{p}<0,01$.

\subsubsection{Norme}

Presenteremo di seguito le medie e le deviazioni standard delle diverse scale della prova, per i 4 e i 5 anni (vd. Tabelle 4 e 5).

Le norme sono state calcolate per $1 / 3$ con soggetti di livello medio e per $2 / 3$ su campioni di soggetti in ambiente socio-culturale medio-basso.

Questi ultimi sono contesti con le maggiori carenze di readiness e in cui è più urgente intervenire per sviluppare i requisiti di readiness. 
Tabella 4. - Norme per i 4 anni per le singole scale $(n=111)$.

\begin{tabular}{rrrrrrrrr}
\hline & $\begin{array}{c}\text { Mem. } \\
\text { vis. }\end{array}$ & $\begin{array}{l}\text { Mem. } \\
\text { Verb. }\end{array}$ & Conos. & Compr. & Ragion. & $\begin{array}{l}\text { Cap. } \\
\text { Crit. }\end{array}$ & Creativ. & Conteg. \\
\hline m & 5,30 & 0,97 & 10,66 & 14,52 & 2,82 & 5,51 & 5,30 & 3,16 \\
d.s. & 2,59 & 0,82 & 4,26 & 5,04 & 1,20 & 3,03 & 3,69 & 1,25 \\
\hline
\end{tabular}

Tabella 5. - Norme per is anni per le singole scale $(n=334)$.

\begin{tabular}{ccccccccc}
\hline & $\begin{array}{c}\text { Mem. } \\
\text { vis. }\end{array}$ & $\begin{array}{c}\text { Mem. } \\
\text { Verb. }\end{array}$ & Conos. & Compr. & Ragion. & $\begin{array}{c}\text { Cap. } \\
\text { CRit. }\end{array}$ & Creativ. & Conteg. \\
\hline $\mathrm{m}$ & 5,12 & 1,25 & 8,77 & 17,12 & 4,28 & 3,81 & 6,22 & 5,63 \\
d.s. & 2,86 & 1,04 & 3,33 & 4,27 & 1,57 & 2,68 & 3,86 & 2,15 \\
\hline
\end{tabular}

\subsection{Riflessioni critiche}

La difficoltà di utilizzo della scala da parte degli insegnanti è legata alla necessità di somministrazione individuale, che richiede da 30 a 40 minuti, a seconda del livello d'età dei bambini. La scala presuppone inoltre un'adeguata formazione degli insegnanti sui fattori che possono interferire nella somministrazione, sulle condizioni da rispettare per la stessa e sui processi cognitivi che vengono rilevati, per poter interpretare correttamente i dati e strutturare interventi di mediazione cognitiva successivi efficaci. Per il bambino di recente migrazione le interferenze linguistiche nello svolgimento della prova possono portare a sottovalutare il potenziale di apprendimento. In tal caso occorrerebbe avvalersi della mediazione linguistica, affiancata da una rilevazione ad hoc per gli aspetti linguistici. Lo strumento richiede inoltre adattamenti nel caso venga utilizzato in contesti culturali molto distanti da quello italiano, come avviene abitualmente per i test non culture free.

\section{Conclusione}

Il presente contributo ha evidenziato la rilevanza di una misura adeguata di readiness per diagnosi precoci, finalizzate ad attivare interventi tempestivi, così da favorire la riuscita dei bambini nella scuola dell'infanzia e nei gradi 
scolastici successivi. Tali rilevazioni sono necessarie anche quando mancano professionisti che possono somministrare test. Occorrono in tal caso sussidi validi e affidabili, ma adatti alla professionalità degli insegnanti. Per rispondere a questa esigenza abbiamo elaborato uno strumento, non certo a fini selettivi o per l'individuazione di bisogni educativi speciali, ma per mettere le premesse per il successo scolastico successivo, attraverso diagnosi adeguate e l'adozione di coerenti misure compensative, nei casi di difficoltà. Come ha sottolineato un'importante ricerca evidence based, attraverso meta-analisi su 931 ricerche empiriche, la formazione ricevuta nella scuola dell'infanzia è significativamente rilevante per gli apprendimenti successivi (Hattie, 2012). L'insegnante può infatti, con azioni specifiche, prevenire difficoltà di apprendimento nella scuola primaria, attivando percorsi ludici volti a potenziare i processi cognitivi risultati carenti, sostenendo e rinforzando la motivazione ad apprendere. A questo proposito, risultati di ricerca (Ricchiardi \& Coggi, 2011) hanno evidenziato che si può ottenere un incremento significativo nella riuscita media dei bambini in difficoltà, con un percorso ludico a piccolo gruppo, che si avvale della mediazione cognitiva ed affettiva di un docente, e di una valigetta di giochi appositamente predisposta. Le rilevazioni condotte con il test qui presentato hanno evidenziato progressi del $23 \%$ sui gruppi sperimentali, rispetto ad una crescita dell' $8 \%$ dei gruppi di controllo. Studi di follow up confermano il persistere dei progressi conseguiti ad un anno di distanza (Coggi \& Ricchiardi, 2014), confermando il valore di diagnosi precoci seguite da interventi efficaci.

\section{RIFERIMENTI BIBLIOGRAFICI}

Andrews, S. P., \& Slate, J. R. (2001). Prekindergarten programs: A review of the literature. Current Issues in Education, 4(5), online.

Bierman, K. L., Torres, M. M., Domitrovich, C. E., Welsh, J. A., \& Gest, S. D. (2009). Behavioral and cognitive «readiness» for school: Cross-domain associations for children attending head start. Social Development, 18(2), 305-323.

Blair, C. (2006), How similar are fluid cognition and general intelligence? A developmental neuroscience perspective on fluid cognition as an aspect of human cognitive ability. Behavioral and Brain Sciences, 29, 109-160.

Boncori, L. (1993). Teoria e tecniche dei test. Torino: Bollati Boringhieri.

Bornstein, M. H., Hahn, C., \& Haynes, O. (2004). Specific and general language performance across early childhood: Stability and gender considerations. First Language, 24, 267-304. 
Bornstein, M. H. (1995). Parenting infants. In Id. (Ed.), Handbook of parenting, Vol. 1: Children and parenting (pp. 3-39). London: Lawrence Erlbaum Associates.

Bracken, B. A. (2007). Bracken School Readiness Assessment (3rd ed.) (BSRA-3). San Antonio, TX: Harcourt Assessment.

Brickman, N., \& Barton, H. (2003). COR. Observation items. Ypsilanti, MI: High Scope Press.

Chaplin, T. M., Cole, P. M., \& Zahn-Waxler, C. (2005). Parental socialization of emotion expression: Gender differences and relations to child adjustment. Emotion, 5, 80-88.

Coggi, C., \& Ricchiardi, P. (2014). Garantire la «school readiness» di tutti i bambini. In G. Cerrato, C. Sabena, \& E. Scalenghe, L’apprendimento nella scuola dell'infanzia. Riflessioni teoriche ed esperienze didattiche. Roma: Aracne.

Coggi, C., \& Ricchiardi, P. (2009). Gioco e potenziamento cognitive nei contesti deprivati. In R. Quaglia, L. E. Prino, \& E. Sclavo (a cura di), Il gioco nella didattica. Un approccio ludico per la scuola dell'infanzia e primaria (pp. 13-48). Trento: Erickson.

Davis, H. L., \& Pratt, C. (1996). The development of children's theory of mind: The working memory explanation. Australia Journal of Psychology, 47, 25-31.

Deci, E., \& Ryan, R. (1985). Intrinsic motivation and self-determination in human behavior. New York: Plenum.

De Feyter, J. J., \& Winsler, A. (2009). The early developmental competencies and school «readiness» of low-income, immigrant children: Influences of generation, race/ethnicity, and national origins. Early Childhood Research Quarterly, 24, 411-431.

De Lemons, M., \& Doing, B. (2000). Who am I? Camberwell: Acer Press.

Diamond, A., \& Taylor, C. (1996). Development of an aspect of executive control: Development of the abilities to remember what I said and to «do as I say, not as I do". Developmental Psychobiology, 29, 315-334.

Dickinson, D. K., \& Tabors, P. O. (2001). Book reading in preschool classrooms: Is recommended practice common? In O. K. Dickinson \& P. O. Tabors, Beginning literacy with language: Young children learning at home and school (pp. 175-203). Baltimore, MD: Paul H. Brookes.

Duncan, G. J., \& Magnuson, K. A. (2005). Can family socioeconomic resources account for racial and ethnic test score gaps? Future of Children, 15, 35-53.

Duncan, G.J. et al. (2007). School «readiness» and later achievement. Developmental Psychology, 43(6), 1428-1446.

Dunn, L. M., \& Dunn, D. M. (2000). PPVT-4. Toronto: Pearson Education (trad. it. a cura di G. Stella, C. Pizzoli, \& P. Tressoldi, Peabody - Test di Vocabolario Recettivo (PPVT-R). Torino: Omega). 
Edwards, B., Baxter, J., Smart, D., Sanson, A., \& Hayes, A. (2009). Financial disadvantage and children's school «readiness». Family Matters, 83, 23-31.

Field, T. (1995). Psychologically depressed parents. In M. H. Bornstein (Ed.). Handbook of parenting (pp. 85-100). Mahwah, NJ: Erlbaum.

Frank Porter Graham Child Development Center (1999). Making the transition. Early Developments, 3(1), 4-6.

Frey, D., \& Zelazo, P. D. (2003). Theory of mind and rule-based reasoning. Cognitive Development, 10, 483-527.

Gesell, A., \& Amatruda, S. (1947). Developmental diagnosis (2nd ed.). New York: Hoeber-Harper.

Gibbs, S. (2004). Phonological awareness: An investigation into the developmental role of vocabulary and short-term memory. Educational Psychology, 24(1), 13-25.

Gioia, G. A., Isquit, P. K., Guy, S., \& Kenworthy, L. (2000). Test review. Behavior rating inventory of executive function. Child Neuropsychology, 6(3), 235-238.

Goodman, R. (1997). The Strengths and Difficulties Questionnaire: A research note. Journal of Child Psychology and Psychiatry, 38, 581-586.

Goodman, R. (2001). Psychometric properties of the Strengths and Difficulties Questionnaire (SDQ). Journal of the American Academy of Child and Adolescent Psychiatry, 40, 1337-1345.

Grant, D. A., \& Berg, E. A. (1948). A behavioral analysis of degree of reinforcement and ease of shifting to new responses in a Weigl-type card-sorting problem. Journal of Experimental Psychology, 38, 404-411.

Halle, T., Forry, N., Hair, E., Perper, K., Wandre, L., Wessel, J., \& Vick, J. (2009). Disparities in early learning and development lesson from the Early Childhood Longitudinal Study-Birth cohort (ECLS-B). Washington, DC: Child Trends.

Hart, B., \& Risley, T. R. (1995). Meaningful differences in the everyday experience of young american children. Baltimore, MD: Paul H. Brookes Publishing Co.

Hattie, J. (2012), Visible learning for teachers. Maximizing impact on learning, London New York: Routledge.

Hibel, J. (2009). Root of assimilation. Generational status differentials in ethnic minority children's school «readiness». Journal of Early Childhood Research, 7(2), 135-152.

Howard, T., Grogan Dresser, S., \& Dunklee, D. R. (2009). Poverty is not a learning disability. Thousand Oaks, CA: Corwin.

Huffman, L. C., Mehlinger, S. L., \& Kerivan, A. S. (2001). Off to good start: Research on the risk factors for early school problems and selected federal policies affecting children's social and emotional development and their "readiness" school. Chapel Hill, NC: Frank Porter Graham Child Development Center. 
Janus, M. (2007). The early development instrument: A population-based measure for communities. A handbook on development, properties, and use. Oxford: Center for Child Studies.

Kagan, S. L., \& Rigby, E. (2003). Policy matters: Setting and measuring benchmarks for state policies. Washington, DC: Center for the Study of Social Policy.

Kagan, S. L., Moore, E., \& Bredekamp, S. (1995). Reconsidering children's early development and learning. Toward common views and vocabulary. Washington, DC: National Education Goals Panel.

Keating, D. P. (2007). Formative evaluation of the early development instrument: Progress and prospects. Early Education and Development, 18(3), 561-570.

Keenan, K., Shaw, D. S., Walsh, B., Delliquadri, E., \& Giovannelli, J. (1997). DSM-III disorders in preschool children from low income families. Journal of American Academy of Child and Adolescent Psychiatry, 36, 620-627.

Kenny, A. (1978). The development of a diagnostic instrument based on a Piagetian model. Nova, FL: Nova University.

Kiernan, G. et al. (2008). The school «readiness» of children living in a disadvantaged area in Ireland. Journal of Early Childhood Research, 6(2) 119-144.

Kochanska, G., Murray, K., Jacques, T. Y., Koenig, A. L., \& Vandegeest, K. A. (1996). Inhibitory control in young children and its role in emerging internalization. Child Development, 67(2), 490-507.

Ladd, G. W., \& Burgess, K. (1999). Charting the relationship trajectories of aggressive, withdrawn, and aggressive/withdrawn children during early grade school. Child Development, 70(4), 910-929.

Ladd, G. W., Kochenderfer, B. J., \& Coleman, C. C. (1997). Classroom peer acceptance, friendship, and victimization: Distinct relational systems that contribute uniquely to children's school adjustment. Child Development, 68(6), 1181-1197.

La Paro, K. M., \& Pianta, R. C. (2000). Predicting children's competence in the early school years: A meta-analytic review. Review of Educational Research, 70(4), 443-484.

Loeb, S., Bridges, M., Bassok, D., Fuller, B., \& Rumberger, R. W. (2007). How much is too much? The influence of preschool centres on children's social and cognitive development. Economics of Education Review, 26, 52-66.

Love, J. M., Aber, L., \& Brooks-Gunn, J. (1994). Strategies for assessing community progress toward achieving the first national educational goal. Princeton: Mathematical Policy Research.

Magnuson, K. A., Meyers, M. K., Ruhm, C. J., \& Waldfogel, J. (2004). Inequality in preschool education and scholl «readiness». American Educational Research Journal, 41(1), 115-157. 
Mashburn, A. J., \& Henry, G. T. (2004). Assessing school «readiness»: Validity and bias in preschool and kindergarten teachers' ratings. Educational Measurement: Issues and Practice, 23(4), 16-30.

Maxwell, K. L., \& Clifford, R. C. (2004). Research in review: School «readiness» assessment. Young Children, 59(1), 42-46.

McCartney, K. et al. (2007). Quality child care supports the achievement of lowincome children: Direct and indirect pathways through caregiving and the home environment. Journal of Applied Development Psychology, 28, 411-426.

Meisels, S. J. (1999). Assessing «readiness». In R. C. Pianta \& M. Cox (Eds.), The transition to kindergarten (pp. 39-66). Baltimore, MD: Paul Brookes.

National Center for Education Statistics (1993). Public school kindergarten teachers' views on children's readiness for school. Washington, DC: Department of Education, Office of Educational Research and Improvement.

Niemeyer, J., \& Scott-Little, C. (2001). Assessing kindergarten children: A compendium of assessment instrument. Greensboro, NC: SERVE.

Nurss, J. R., \& McGauvran, M. (1976). Metropolitan Readiness Tests - Fourth Edition, San Antonio, TX: The Psychological Corporation.

Pianta, R. C., \& Walsh, D. J. (1998). Applying the construct of resilience in schools: Cautions from a developmental systems perspective. School Psychology Review, 27, 407-417.

Portes, A., \& Rumbaut, R. (2000). Legacies: The story of the immigrant second generation. Berkeley, CA: University of California Press.

Raver, C. C., \& Knitze, J. (2002). Promoting the emotional well-being of children and families policy. Paper 3: Ready to enter: What research tells policymakers about strategies to promote social and emotional school readiness. Among three- and four-year-old children (pp. 1-24). New York: Columbia University, National Center for Children in Poverty.

Raver, C. C., \& Zigler, E. F. (1997). Social competence: An untapped dimension in evaluating head start's success. Early Childhood Research Quarterly, 12(4), 363-385.

Ricchiardi, P., \& Coggi, C. (2011). Gioco e potenziamento cognitivo nell'infanzia. La teoria. Trento: Erickson.

Robertson, C., \& Salter, W. (1997). The Phonological Awareness Test. East Moline, IL: LinguiSystems.

Sanford, A. R., \& Zelman, J. G. (2003). Test LAP. Diagnosi di sviluppo. Trento: Erickson.

Schuele, C. M. (2004). The impact of developmental speech and language impairments on the acquisition of literacy skills. Mental Retard Development Disability. Research Review, 10(3), 176-183.

Shanahan, T. (2005). The national reading panel Report. Practical advice for teachers. Naperville, IL: Learning Point Association. 
Sylva, K. (2002). The role of research in explaining the past and shaping the future. In L. Abbott \& H. Moylett (Eds.), Early education transformed (pp. 164179). London: Routledge Falmer.

Tran, H., \& Weinraub, M. (2006). Childcare effects in context: Quality, stability, and multiplicity in nonmaternal child care arrangements during the first 15 months of life. Developmental Psychology, 42, 566-582.

Webster-Stratton, C., Reid, M. J., \& Stoolmiller, M. (2008). Preventing conduct problems and improving school «readiness»: Evaluation of the incredible years teacher and child training programs in high-risk schools. Journal of Child Psychology and Psychiatry, 49(5), 471-488.

Woodcock, R. W., Mather, N., McGrew, S. K. (2001). Woodcock-Johnson III Tests of Cognitive Abilities. Itasca, IL: Riverside.

Zanetti, M. S., \& Miazza, D. (2003). Test SR 4-5 (School Readiness 4-5 anni). Prove per l'individuazione delle abilità di base nel passaggio dalla scuola dell'infanzia alla scuola primaria. Trento: Erickson.

Zill, N., \& West, J. (2001). Findings from the condition of education 2000: Entering kindergarten (Rep. No. NCES 2001035). Washington, DC: U.S. Department of Education.

\section{Riassunto}

Molti studi a livello internazionale si sono focalizzati sulla «school readiness». È diventato dunque un tema su cui è urgente effettuare bilanci delle evidenze empiriche. Si tratta di una caratteristica complessa, che ha dato origine a un ampio dibattito e a una varietà di apporti. Questi ne hanno approfondito il concetto e gli ambiti da indagare. Hanno evidenziato inoltre le condizioni ambientali che concorrono allo sviluppo della «readiness» e le possibili strategie di potenziamento. Il presente contributo effettua un bilancio critico di tali studi. Il saggio si focalizza in particolare sugli aspetti della misurazione della "readiness» e offre una rassegna dei principali test adottati internazionalmente per misurarla. Propone infine uno strumento originale di misura della "readiness» cognitiva, adatto alle scuole italiane, e ne descrive le caratteristiche psicometriche, calcolate su un campione di 445 bambini. Tale prova consente di diagnosticare le difficoltà che percentuali significative di bambini presentano tra $i 4$ e i 5 anni e permette di valutare gli effetti di interventi di potenziamento della "readiness", cosi da individuare quelli più efficaci, secondo la prospettiva dell'EBE.

Parole chiave: Modelli di readiness, Preparazione scolastica iniziale, Prontezza scolastica, Scuola dell'infanzia, Test di readiness, Valutazione della readiness. 